Tropical Journal of Pharmaceutical Research January 2017; 16 (1): 239-244

ISSN: $1596-5996$ (print); 1596-9827 (electronic)

(c) Pharmacotherapy Group, Faculty of Pharmacy, University of Benin, Benin City, 300001 Nigeria.

All rights reserved.

Available online at http://www.tjpr.org

Original Research Article

http://dx.doi.org/10.4314/tjpr.v16i1.32

\title{
Detection of drug-induced dyslipidaemia in HIV-positive patients treated with protease inhibitors in a South African district $-A$ retrospective study
}

\author{
Pierre Mugabo ${ }^{1^{*}}$ and Richard Madsen ${ }^{2}$ \\ ${ }^{1}$ School of Pharmacy, University of the Western, Cape Town, South Africa, ${ }^{2}$ Department of Statistics, University of Missouri, \\ USA
}

*For correspondence: Email: pmugabo@uwc.ac.za; Tel: +27-219592190

Received: 13 September 2016

Revised accepted: 19 December 2016

\begin{abstract}
Purpose: To determine whether protease inhibitors (PIs) cause hypercholesterolaemia and hypertriglyceridaemia, and to assess the influence of sex and age on serum total cholesterol (TC) and triglycerides (TG), and the level of adherence to therapeutic laboratory monitoring guidelines in HIV positive patients in the Eastern Health District, South Africa.

Methods: This was a retrospective study which compared baseline and follow-up TC and triglycerides serum levels in HIV-infected patients who received treatment, including PIs, between 2008 and 2012 in the Eastern Health District of the Cape Metropole Region (Cape Town, South Africa). Clinical and laboratory data were retrieved from patients' folders and from a computerized database at National Health Laboratory Services (NHLS). The level of compliance with therapeutic laboratory monitoring protocols by health workers and patients was also assessed.

Results: Out of 753 patients (491 females, 262 males), 176 were 0 - 17, 465 were 18 - 44 and 12 were $>44$ years old. The proportion of patients who had test results at the various time points ranged from 16.33 to $25.90 \%, 17.66$ to $24.83 \%, 25.10$ to $47.41 \%$ and 25.23 to $36.79 \%$ for TC, triglycerides, CD4 count, and viral load (VL) tests, respectively. There was a significant time increase $(p=0.0137)$ for serum TC from baseline to 3 years. For serum triglycerides, the overall time effect was not significant ( $p$ $=0.4132$ ). There was a significant increase from baseline in CD4 and a significant decrease in VL during the study period. Sex and age did not show any relationship with TC and triglyceride serum levels.

Conclusion: This study demonstrated hypercholestrolaemia in HIV-infected patients receiving PIs. Age and sex did not have any effect on TC and triglycerides. Compliance with therapeutic laboratory monitoring guidelines was poor.
\end{abstract}

Keywords: Hyperlipidaemia, Protease inhibitors, Therapeutic monitoring, HIV infection, Hypercholesterolaemia, Hypertriglyceridaemia

Tropical Journal of Pharmaceutical Research is indexed by Science Citation Index (SciSearch), Scopus, International Pharmaceutical Abstract, Chemical Abstracts, Embase, Index Copernicus, EBSCO, African Index Medicus, JournalSeek, Journal Citation Reports/Science Edition, Directory of Open Access Journals (DOAJ), African Journal Online, Bioline International, Open-J-Gate and Pharmacy Abstracts

\section{INTRODUCTION}

Antiretroviral therapy (ART) has improved the prognosis of HIV-infection during the last decade. However, antiretroviral drugs are frequently associated with serious side effects including haematological, gastrointestinal, neurological, mental, skin and metabolic disorders [1].

Studies conducted in developed and developing countries have shown conclusive results that protease inhibitors (PIs) cause hyperlipidaemia [1-4]. About $60 \%$ of HIV-positive patients 
develop hyperlipidaemia due to an abnormal lipid metabolism [5]. Hyperlipidaemia developing in HIV-positive patients may be due to the effect of PIs or HIV infection or the combination of both on the metabolism of lipids [6]. Stavudine and nonnucleotide reverse transcriptase inhibitors (NNRTIs) can also cause abnormal changes in lipid profile, but to a lesser extent than PIs [1].

According to the South African Department of Health (DOH) treatment guidelines, serum TC and serum TG are monitored at baseline, again at three months and then every year after starting Pls treatment. The protocol does not recommend follow-up of low density lipoprotein (LDL), very low density lipoprotein (VLDL) and high density lipoprotein (HDL) - cholesterol levels [7].

During the study period, the second line HIVinfection treatment for children included zidovudine, didanosine and Lopinavir /ritonavir. Children failing on this regimen were placed on abacavir, lamivudine and lopinavir/ritonavir. The second line treatment regimen for adolescents and adults included zidovudine, lamivudine and lopinavir/ritonavir [8].

Lopinavir/ritonavir were given at a fixed dose of 400/200 mg 12 hourly in adults and 230/57.5 $\mathrm{mg} / \mathrm{m}^{2} 12$ hourly in children [9].

The objectives of this study were: To find out whether or not PIs cause hypercholesterolaemia and hypertriglyceridaemia in HIV-positive patients; to determine the influence of sex and age on TC and TG serum levels; and to assess the level of compliance with therapeutic laboratory monitoring protocols recommended by the $\mathrm{DOH}$.

\section{METHODS}

The project was designed as a retrospective study, comparing TC and TG serum levels at baseline, three months and every year for three years after initiating Pls. The study also assessed the level of compliance with therapeutic laboratory monitoring protocols by health workers and patients.

The study was conducted at five HIV clinics and one hospital, conveniently selected, in the Eastern Health District of the Cape Metropole region, South Africa. It involved exclusively HIVpositive patients of all ages and both sexes who were on treatment that included Pls for three years between 2008 and 2012. Patients were excluded from the study if any of the following applied: pregnancy, concurrent treatment with other drugs that can cause dyslipidaemia and concomitant diseases (e.g., diabetes mellitus) which interfere with lipid metabolism.

Clinical and laboratory data were retrieved from patients' folders and from a computerized data base obtained from the National Health Laboratory Services (NHLS). The data collected was: the study participant's age, sex and baseline date (date when Pls treatment was started); serum TC, serum TG, CD4 count and viral load. Abnormal lipid profile was defined in accordance with NHLS guidelines, as: TC $\geq 200$ $\mathrm{mg} / \mathrm{dl} / 5.17 \mathrm{mmol} / \mathrm{L}, \mathrm{TG} \geq 150 \mathrm{mg} / \mathrm{dl} / 1.69$ $\mathrm{mmol} / \mathrm{L}$.

The statistical methods used to address the research questions included a repeated measures analysis of variance to compare later values with baseline values, log transformations (logs) to make the responses closer to normal distributions, and the nonparametric Friedman's test for cases where the log transformation was not adequate for getting the data closer to normal. Since values at later times were supposed to be compared to baseline values, adjustment for multiple comparisons was done using Dunnett's method. Adjusted p-values less than 0.05 were considered significant.

\section{Ethical considerations}

This study was approved by the ethics committee of the University of the Western Cape (Reference no. 12/5/7). Permission to conduct the study was granted by the Western Cape Department of Health (ID no. 10317) and the managers of the hospital /community health centres. It was conducted according to the ethics requirements of the South African Department of Health and the Declaration of Helsinki [10]. The information collected was kept confidential and saved in a safe file which could only be accessed by the researcher. Patient confidentiality and privacy were maintained at all times.

\section{RESULTS}

A total of 753 patients including 491 (65.20\%) females and $262(34.79 \%)$ males were involved in the study. In the age group of 0-17 years there were $176(23.37 \%)$ patients, of whom 106 were females and 70 were males. In the age group 1844 years, there were $465(61.75 \%)$ patients, of whom 322 patients were females and 143 were males. In the age group equal and above 45 years of age, there were $112(14.87 \%)$ patients including 63 females and 49 males. 
The CD4 counts, viral loads, serum TC and serum TG results are summarized in Figure 1, 2, 3 and 4 . Figures 1, 2, 3, and 4 show boxplots with a reference line joining the mean values for each time. The $x$-axis shows the times (baseline, 3 months. 1, 2 and 3 years after the start of Pls treatment. The $y$-axis shows the log values of the CD4 counts (Figure 1), viral loads (Figure 2), serum TC (Figure 3 ) and serum TG (Figure 4).

Out of 753 patients, 59 (7.83\%) and 70 (9.29\%) had respectively serum TC and serum TG higher than normal values at baseline.

At baseline the level of compliance with therapeutic laboratory monitoring protocols recommended by the $\mathrm{DOH}$ were 23.24, 24.04, 47.41 and $36.79 \%$ for serum TC, serum TG, CD4 counts and VL respectively. Three months after the beginning of the treatment, the levels of compliance with therapeutic laboratory monitoring decreased to $16.33,17.66,27.76$ and $27.22 \%$ for serum TC, serum TG, CD4 counts and $V L$ respectively. One year after the start of Pls treatment, the level of compliance increased to $25.90,24.83,33.86$ and $38.25 \%$ for serum TC, serum TG, CD4 counts and VL respectively. However, two years after the beginning of treatment, the level of compliance dropped to 18.99, 19.26, 25.10 and $25.23 \%$ for serum TC, serum TG, CD4 counts and VL, respectively.

For CD4 (Figure 1), a log transformation was used, demonstrating that there were differences from baseline values. All comparisons with baseline values illustrated significant differences. However, examination of the residuals indicated a lack of normality. Consequently Friedman's nonparametric test was done. Based on this test there were significant differences from baseline at all times: 3 months, 1, 2 and 3 years after the start of the treatment.

For viral load (Figure 2), log transformation was used. All comparisons with baseline were significant. However, examination of the residuals indicated a lack of normality. Consequently, Friedman's nonparametric test was done. Based on this test there were significant differences from baseline at all times: 3 months, 1, 2 and 3 years.

For serum TC (Figure 1), there was a significant overall time effect $(p=0.0061)$ with a significant difference from baseline at three years (adjusted $p=0.0137)$.

For serum TG (Figure 2), a log transformation was used. The overall time effect was not significant $(\mathrm{p}=0.4132)$.

The mean values for serum TC at baseline were less than $4.5 \mathrm{mmol} / \mathrm{L}$, and so were within the normal ranges. The values then slightly fall below $4 \mathrm{mmol} / \mathrm{L}$ at three months. From one year onwards during Pls treatment, the values increased minimally above $4 \mathrm{mmol} / \mathrm{L}$ but they were still within the normal ranges.

The mean values for serum TG at baseline (Figure 1) were also within the normal ranges -less than $1.7 \mathrm{mmol} / \mathrm{L}$. After three months of Pls treatment, the values surged above the normal values.

Tests were done to find out if sex and age might be related to the TC and TG values at any time. No significant relationships were seen.

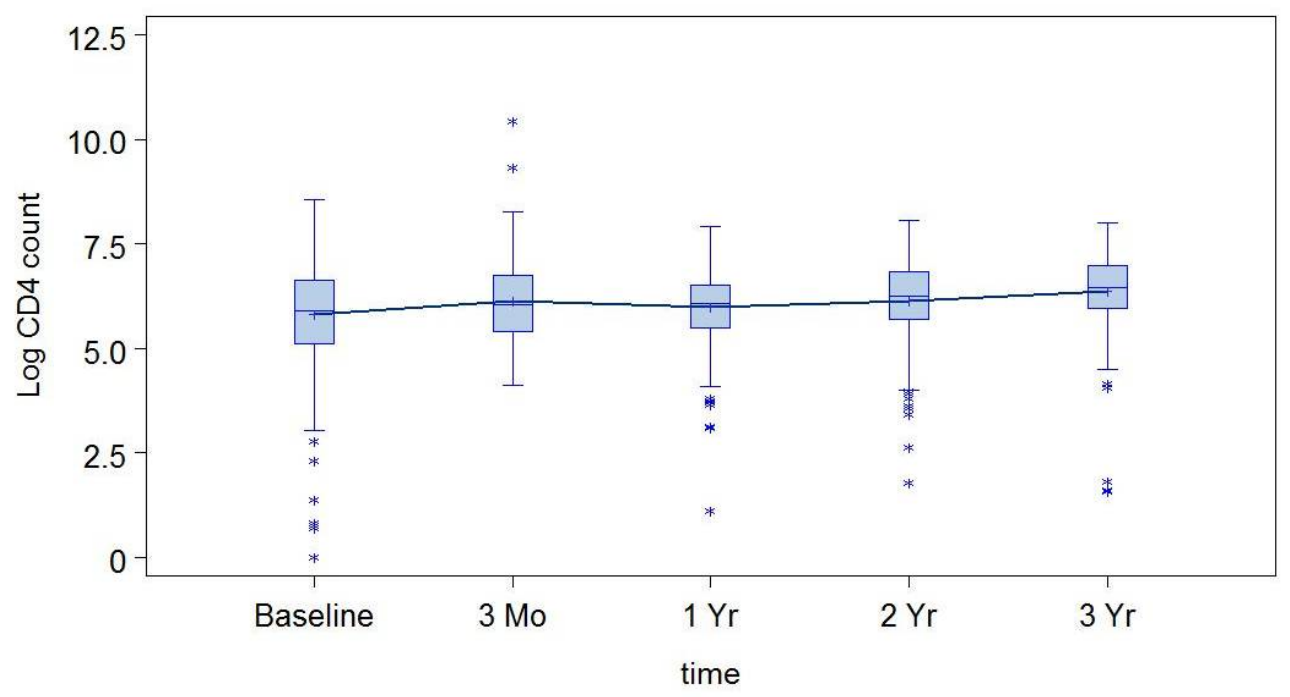

Figure 1: CD4 counts over time 


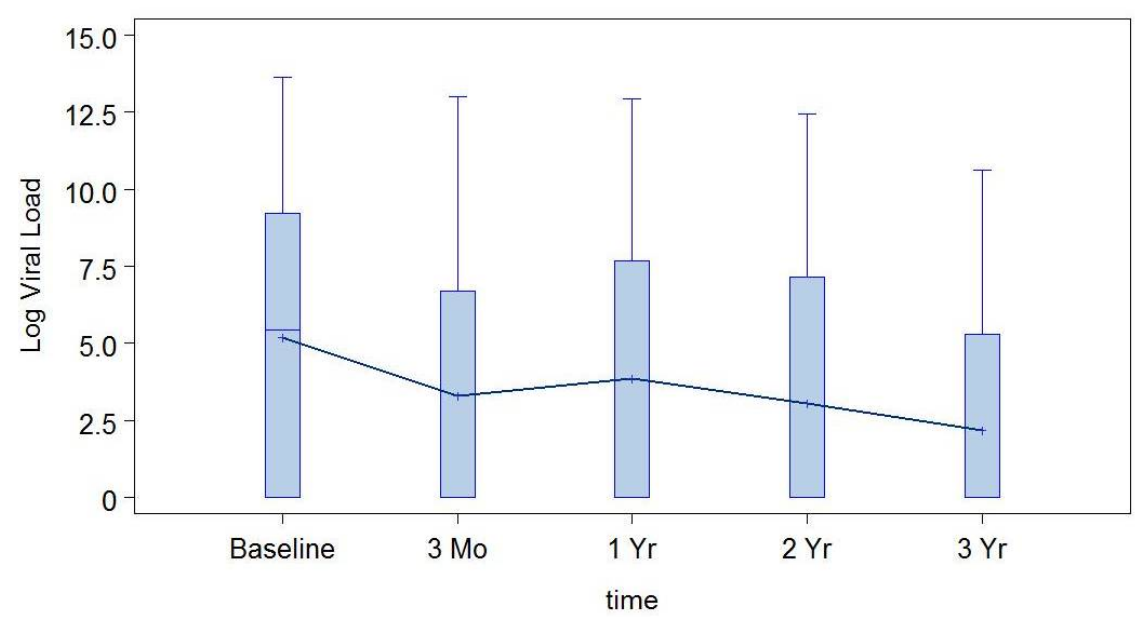

Figure 2: Viral load over time

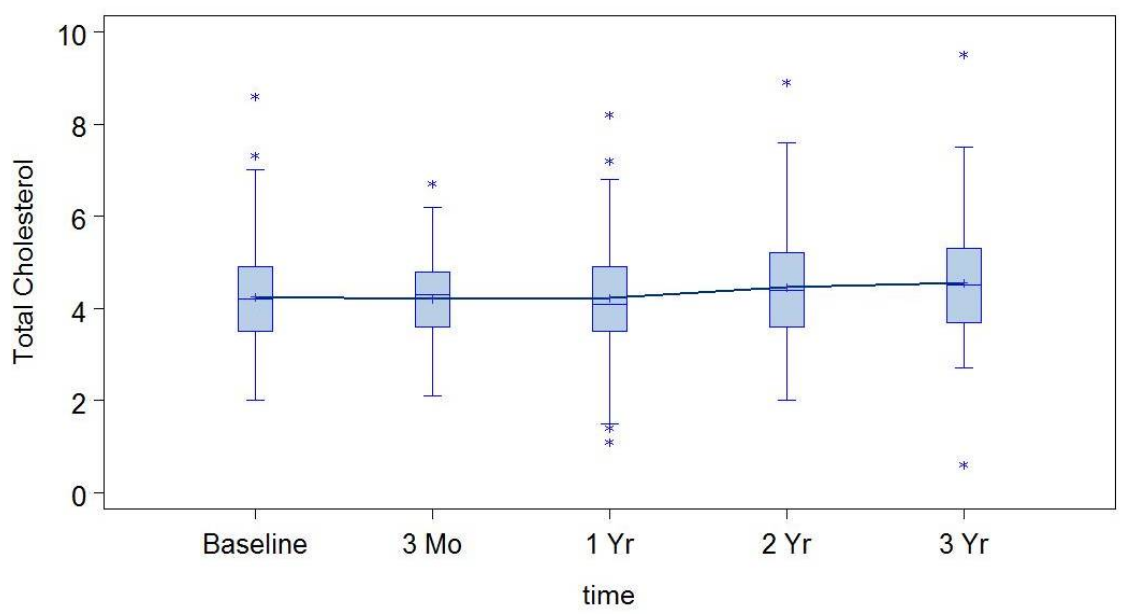

Figure 3: Serum total cholesterol levels over time

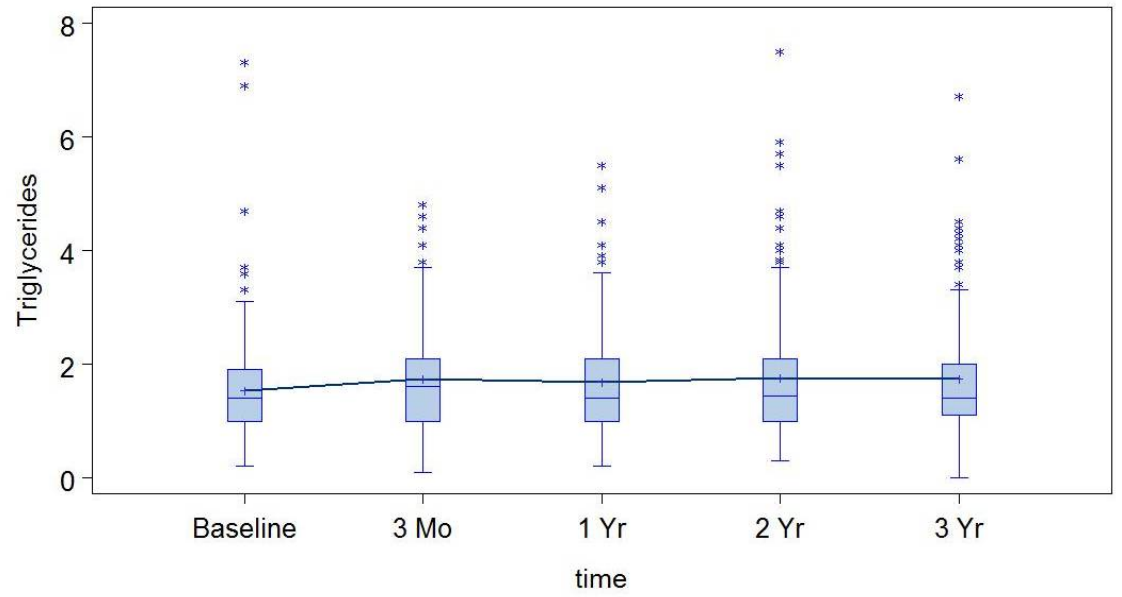

Figure 4: Serum triglyceride levels over time

\section{DISCUSSION}

In this study, female and male patients were well represented in all age ranges.
The high TC and TG serum levels observed at baseline were most likely due to either primary hyperlipidaemia or to previous exposure to drugs (eg. NRTIs in adult patients) that could alter the lipid profile. It could also be due to a decreased 
liver function [1]. Liver function tests results were not available to rule out that possibility.

Previous studies have reported menopause induced reduction of HDL-cholesterol, and elevation of TC, TG, LDL -cholesterol and VLDLcholesterol [11]. In this study, there was no significant difference in TC and TG serum levels between males and females within this age range, suggesting that female hormonal changes did not influence lipid levels.

This study demonstrated a significant difference in TC serum levels between baseline and 3 years. TG serum levels did not significantly change.

According to Carr et al, Pls may not be the only class of antiretroviral medication associated with dyslipidaemia [2]. NNRTI therapy was associated with hypercholesterolemia and a statistically significant linear, temporal increase in serum TC levels. However, the effect of NNRTIs on TC serum levels was weaker than the effect of PIs [2].

The trend indicates that the longer the patient is exposed to Pls, the more CD4 cell counts increase, and viral load decreases. TC serum levels increased, as a consequence of the aforementioned trend. Although treatment with Pls contribute to patient susceptibility to metabolic disorders such as dyslipidaemia, they have also improved the prognosis of HIV infection in the HIV clinics where the study was conducted.

The increase in serum TG levels reported in Figure 1 could be induced by $\mathrm{Pls}$ or due to dietary lifestyles of patients. After one year, the values slightly decreased and this may be as a result of initiation of lipid lowering therapy. After two years, the values moderately increased above normal values and remained constant up to three years of treatment. The results thus indicate that there was no hyperlipidaemia at baseline.

The observed lack of compliance with therapeutic laboratory monitoring guidelines can be attributed, on one side, to health professionals who did not collect blood samples for laboratory tests and, on the other, to patients who did not keep their appointments. Consequently, it will be difficult to detect cases of dyslipidaemia which may lead to severe cardiovascular complications if not treated.

\section{Limitations of the study}

The following unforeseen problems were encountered: poor laboratory results reporting and recording in the patient's folder, poor administration of patient folders, and lack of knowledge of the extent to which 1st line ARV may have induced hyperlipidaemia.

\section{CONCLUSION}

This study demonstrates a significant increase in TC serum levels due to PIs, but increase in TG serum levels is not evident. Age and sex do not have any effect on both TC and TG serum levels. Compliance of patients and/or health care professionals with $\mathrm{DOH}$ protocols on laboratory monitoring of HIV infection therapy is poor.

\section{DECLARATIONS}

\section{Acknowledgement}

The authors would like to acknowledge the management and staff members of Michael Mapongwana and Micthell's Plain Community Health Centres; Ikhwezi, Langa and Delft South HIV clinics; Helderberg Hospital for permission and assistance to access patients'folders; BPharm IV students (University of the Western Cape) who participated in the data collection; the Western Cape Department of Health for permission to conduct the study; the National Health Laboratory Services (NHLS) for access to laboratory test results and the University of the Western Cape for financial support.

\section{Conflict of Interest}

No conflict of interest associated with this work.

\section{Contribution of Authors}

The authors declare that this work was done by the authors named in this article and all liabilities pertaining to claims relating to the content of this article will be borne by them.

\section{Open Access}

This is an Open Access article that uses a funding model which does not charge readers or their institutions for access and distributed under the terms of the Creative Commons Attribution License (http://creativecommons.org/licenses/by 14.0) and the Budapest Open Access Initiative (http://www.budapestopenaccessinitiative.org/rea d), which permit unrestricted use, distribution, 
and reproduction in any medium, provided the original work is properly credited.

\section{REFERENCES}

1. Yone EWP, Kengne AP, Ashuntantang $G$, Betyoumin $A F$, Ngongang J. Dyslipidaemia in HIV-1-infected patients receiving protease inhibitors after initial treatment with first-line-based non-nucleoside reverse transcriptase inhibitors: a cross-sectional study. BMJ Open 2012; 2(4): e001317 doi:10.1136/bmjopen-2012-001317

2. Carr $A$, Samaras $K$, Chisholm D, Cooper DA. Pathogenesis of HIV-1 protease inhibitor - associated peripheral lipodystrophy, hyperlipidemia and insulin resistance. Lancet 1998; 351: 1881-1883.

3. Tsierras S, Mantzoros C, Hammer S, Samore M. Effects of Protease Inhibitors on Hyperglycemia, Hyperlipidemia, and Lipodystrophy. A 5-Year Cohort Study. Arch Intern Med 2000; 160: 2050-2056

4. Hiransuthikul N, Hiransuthikul P, Kanasook Y. Lipid profiles of Thai adult HIV-infected patients receiving protease inhibitors. South-east Asian J Trop Med Public Health 2008; 38(1): 69-77.
5. Stein JH. Dyslipidaemia in the Era of HIV Protease Inhibitors. Progress in Cardiovascular Diseases 2003; 45(4): 293-304

6. Hui DY. HIV protease inhibitors and atherosclerosis. $J$ Clin Invest 2003; 111: 317-318 doi:10.1172/ JCl200317746.

7. Department of Health. Republic of South Africa. Clinical guidelines for the management of HIV \& AIDS in adults and adolescents. 2010

8. Department of Health. Republic of South Africa. User Guide-UPFS 2009.

9. Gibbon JC. South African Medicines Formulary, 8th edn. Cape Town: South African Medical Association 2008; pp 327-328

10. World Medical Association Declaration of Helsinki: Ethical principles for medical research involving human subjects. JAMA 2013; 310 (20): 2129-4. Doi: 10.1001/Jama. 2013. 281053.

11. Srinivas RK, Srinivasa RC. A Comparative Study of Lipid Profile and Oestradiol in Pre- and Post-Menopausal Women. J Clin Diagn Res 2013; 7(8): 1596-1598. doi: 10.7860/JCDR/2013/6162.3234 